\title{
具有选择性抗癌活性的含硒配位组装体：活性氧物种的调控
}

\author{
李天予易宇许华平* \\ (有机光电子与分子工程教育部重点实验室 清华大学化学系 北京 100084)
}

\begin{abstract}
摘要 活性氧物种(ROS)由于其在生物体内的 “双刃剑” 作用受到越来越多的关注. ROS 在低浓度下能够促进细胞生长, 而在高浓度下会诱导细胞调亡. 硒作为人体必需的微量元素之一, 具有调控细胞内 ROS 浓度的作用. 通过研究具有不 同结构的含硒两亲性分子与顺铂、二氯化铂的配位情况, 探讨硒与铂之间的配位作用; 并进一步研究配位组装体在细 胞内调控 ROS 的能力, 探讨选择性抗癌活性的产生机制. 结果表明, 含硒分子能够与铂类化合物进行配位, 生成具有 选择性抗癌活性的组装体; 该抗癌活性源于组装体对细胞内 ROS 浓度的调控. 期望能够拓展含硒配位组装体在抗癌领 域的应用, 为选择性抗癌药物的开发提供新的思路.
\end{abstract}

关键词 硒; 自组装; 活性氧物种; 配位; 抗癌

\section{Selenium-containing Coordinating Assemblies with Selective Anti-cancer Activity: the Control of Reactive Oxygen Species}

\author{
$\mathrm{Li}$, Tianyu Yi, Yu Xu, Huaping* \\ (Key Lab of Organic Optoelectronics \& Molecular Engineering, Department of Chemistry, \\ Tsinghua University, Beijing 100084)
}

\begin{abstract}
Reactive oxygen species (ROS) attract increasing interests due to the function of "double-edged sword" in physiological condition. In low concentration, ROS can benefit the growth of normal cells; however, in high concentration, they will induce cell apoptosis. Selenium is an essential trace element of human beings that can regulate the concentration of ROS. Previous work demonstrated the selective anti-cancer activity of coordinating assemblies consisting of selenium-containing amphiphile (EGSe) and cisplatin, but the structure and mechanism remain unclear. We synthesized another selenium-containing amphiphile-trimethyl-EGSe (EGSe-tMe) which has similar structure with EGSe but forms weaker interaction with platinum-containing compounds. Then, we prepared new coordinating assemblies composed of two types of selenium-containing amphiphiles and two types of platinum-containing compounds (cisplatin, platinum dichloride), in order to study the coordinating interaction between selenium and platinum. Additionally, we studied the mechanism of their selective anti-cancer activity by testing the ability of regulating ROS concentration in cancer cells and normal cells. We found that EGSe could coordinate with both cisplatin and platinum dichloride, resulting in the formation of coordinating assemblies with selective anti-cancer activity. However, coordinating assemblies consisting of EGSe-tMe and platinum-containing compounds showed little selectivity. These results indicate the important role of tight interaction between selenium and platinum played in the selective anti-cancer activity. The anti-cancer activity comes from their function of regulating the concentration of ROS in cells. Selenium-containing coordinating assemblies can raise higher concentration of ROS in cancer cells than in normal cells, thus causing selective toxicity to cancer cells. In further study, we proposed the mechanism of ROS production by selenium-containing coordinating assemblies. The coordinating assemblies can induce ROS production with the proof of electron spin resonance (ESR) measurement. We hope that this work can not only broaden the applications of selenium-containing coordinating assemblies in the field of cancer treatment, but also provide a new route for developing selective anti-cancer drugs.

Keywords selenium; self-assembly; reactive oxygen species; coordination; anti-cancer
\end{abstract}

\section{1 引言}

近些年来, 活性氧物种(ROS)逐渐受到越来越广泛 的关注, 同时也引发了一些争论. Collins 等 ${ }^{[1]}$ 的研究表 明, ROS 在抗生素诱导细胞凋亡的过程中发挥着重要作
用. 而 Imlay 小组和 Lewis 小组 ${ }^{[2,3]}$ 随后分别对此观点进 行反驳, 认为抗生素诱导细胞调亡的过程与 ROS 无关. ROS 包括氢氧自由基、超氧离子、过氧化氢、单线态氧 等等，它在生物体内具有一种特殊的“双刃剑”作用：低

*E-mail: xuhuaping@mail.tsinghua.edu.cn; Tel.: 0086-010-62773672

Received August 29, 2014; published September 26, 2014.

Project supported by the Foundation for Innovative Research Groups of the National Natural Science Foundation of China (No. 21121004), the Research Project of Chinese Ministry of Education (No. 113006A) and Tsinghua University Initiative Scientific Research Program (No. 2012Z02131).

项目受国家自然科学基金(No. 21121004)、教育部科学技术(No. 113006A)和清华大学自主科研基金(No. 2012Z02131)资助. 
浓度的 ROS 在许多信号传导通路中发挥着至关重要的 作用, 对细胞生长增殖具有促进作用; 而高浓度的 ROS 能够诱导细胞的衰老和调亡, 对细胞产生杀伤作用 ${ }^{[4]}$. 因此, 调控细胞内 ROS 的浓度具有重要的生物学意义.

硒作为人体必需的微量元素之一参与许多生理过 程, 其中非常重要的一项是对人体内氧化还原过程的调 控, 该功能在抗癌方向具有重大应用潜力 ${ }^{[5 \sim 10]}$. 研究表 明含硒分子能够在肿瘤细胞中催化 ROS 的生成, 当 ROS 达到一定浓度时对肿瘤细胞具有杀伤作用 ${ }^{[1]}$. 近 些年来, 科研工作者们报道了一系列含硒高分子构建的 氧化还原响应性药物运输系统, 在抗癌药物的靶向释放 领域取得一定进展 ${ }^{[12 ~ 23] . ~}$

选择性是靶向药物释放领域的核心问题之一, 将药 物定向地释放到目标位点在临床上具有重大意义. 常用 抗癌药物通常不加选择地将正常细胞与癌细胞一同消 灭, 导致严重的毒副作用 ${ }^{[2]}$. 因此, 抗癌药物的剂量受 到严格限制, 在降低毒副作用的同时也大大降低了药物 对癌细胞的作用. 尽管人们已经意识到提高药物的选择 性可以在保证抗癌活性的基础上降低毒副作用, 但癌症 的种类繁多, 而不同患者体内癌细胞周边环境不尽相 同. 所以, 如何实现对癌细胞的高选择性杀灭依然是抗 癌领域的一大难题 ${ }^{[25]}$. 靶向药物运输体系能够有效提 高药物释放的选择性, 因而在近些年中成为该领域热点 之一. 研究者们为实现高选择性的抗癌活性构建了许多

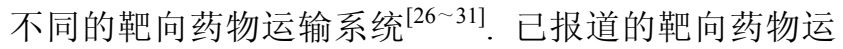
输系统可大体分为两种类型：(1)被动靶向系统，通过 EPR(Enhanced Permeability and Retention)作用实现高选 择性药物释放; (2)主动靶向系统, 利用配体对受体的识 别作用定向释放药物 ${ }^{[32]}$. 虽然已有的靶向药物运输体 系使药物释放的选择性得到一定提升, 但是对正常细胞 的毒副作用依然是制约其发展的一个主要问题, 对该领 域的研究具有广阔前景.

我们近期研究发现, 含硒两亲性分子 EGSe 与顺铂 配位形成的组装体能够高选择性地杀伤癌细胞, 在杀灭 $90 \%$ 以上癌细胞的同时对正常细胞的毒副作用较小 ${ }^{[33]}$. 虽然顺铂本身是一种应用广泛的抗癌药物, 但是其对正 常细胞毒性较大, 并没有显示出明显的选择性. 因此, 我们认为含硒分子对配位组装体的高选择性抗癌活性 起到了十分重要的作用, 对该配位组装体中硒与铂的配 位作用和选择性抗癌机制的研究具有重要意义.

本文证明了结构简单的铂类化合物能够与含硒分 子形成配位组装体, 进而表现出选择性抗癌活性(图 1). 本文对含硒小分子和铂的配位作用以及自组装行为进 行了详细的研究. 同时, 通过测试组装体在细胞内调控 ROS 的能力以及对癌细胞的选择性杀伤作用, 对含硒 配位组装体诱导 ROS 产生的机制进行研究, 探讨选择 性抗癌活性的产生机制. 这一研究提升了硒铂配位体系 的普适性，对构建更多基于硒铂配位作用的药物体系具
有借鉴意义.

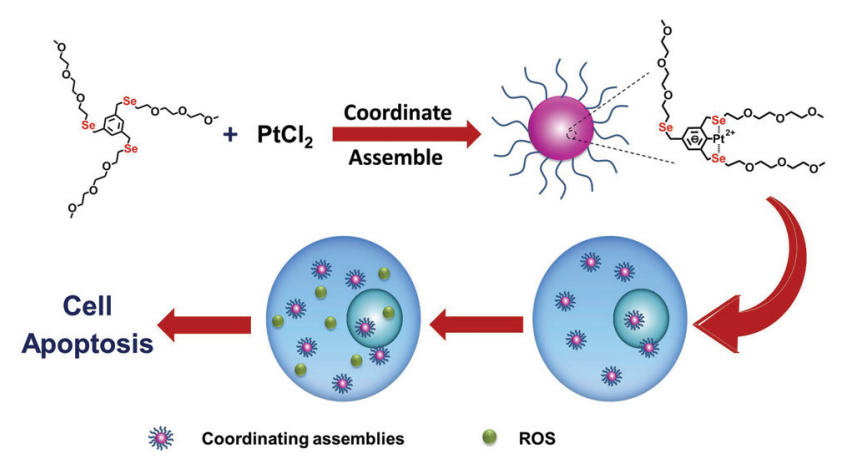

图 1 含硒配位组装体的形成及抗癌机制

Figure 1 Formation and anti-cancer mechanism of selenium-containing coordinating assemblies

\section{2 结果与讨论}

\section{1 硒与铂配位作用的研究}

\subsection{1 顺铂对配位组装体必要性的探讨}

我们近期工作中将含硒分子 EGSe 与顺铂配位，并 表征生成含硒配位组装体 $\mathrm{EGSe} / \mathrm{Pt}$ 的结构(图 1) ${ }^{[33]}$. 由 该结构可知, 顺铂在与含硒分子发生配位作用后失去了 其原有的 4 个配体. 因此, 我们猜测顺铂在配位体系中 的作用可能只是提供铂原子与硒发生配位, 而并不是必 需的. 我们用二氯化铂代替顺铂与 EGSe 进行配位, 对 生成的配位组装体进行 ${ }^{1} \mathrm{H}$ NMR 和 MALDI-TOF 表征 (图 2a, b, S1). ${ }^{1} \mathrm{H}$ NMR 谱图中苯环上氢的吸收峰为 $\delta$ $6.90(\mathrm{~s}, 2 \mathrm{H})$, 乙二醇链段端甲基上氢的吸收峰裂分为双 峰, 与 $\mathrm{EGSe} / \mathrm{Pt}$ 谱图相吻合, MALDI-TOF 得到组装体分 子量为 991.01 , 与 $\mathrm{EGSe} / \mathrm{Pt}$ 的计算值 991.08 近似，说明 EGSe 与二氯化铂生成的配位组装体结构与 EGSe/Pt 相 同. 之后, 我们通过动态光散射(DLS)和透射电子显微 镜(TEM)对 EGSe 与二氯化铂生成的配位化合物的组装 行为进行表征(图 2c, d), 该化合物在水中能够形成直径 为 $100 \mathrm{~nm}$ 左右的球状聚集体, 其组装行为也和 $\mathrm{EGSe} / \mathrm{Pt}$ 相似. 以上实验数据说明 $\mathrm{EGSe}$ 与顺铂或 $\mathrm{PtCl}_{2}$ 进行配 位, 生成的组装体具有相同结构 $\mathrm{EGSe} / \mathrm{Pt}$, 因而顺铂在 配位体系中并不是必需的.

\subsubsection{EGSe-tMe/Pt 配位作用的研究}

为进一步探究硒与铂之间的配位作用情况，我们在 EGSe 中苯环的 2, 4, 6 位分别引入一个甲基, 设计合成 了新型含硒分子 EGSe-tMe(图 3a, S2, S3). EGSe 中苯环 上的氢原子被甲基取代, 因而铂原子无法使苯环上的氢 原子离去, 进而与苯环上的碳原子发生配位. 用 EGSe-TMe 与二氯化铂进行配位，可能导致不同的配位 作用形成.

因为 EGSe-tMe 中苯环部分具有疏水性，而三臂上 的三乙二醇单甲醚链段具有亲水性, 所以该分子具有两 亲性, 在水中能够形成聚集体, 其组装行为的测定结果 


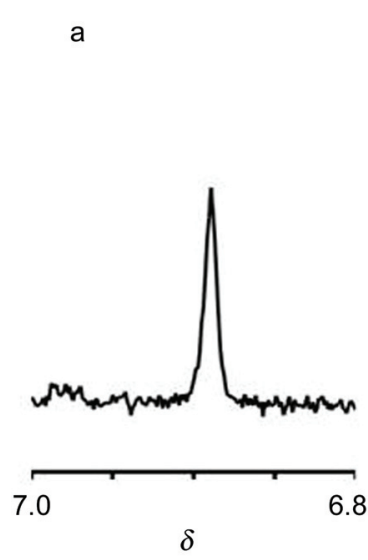

$\delta$

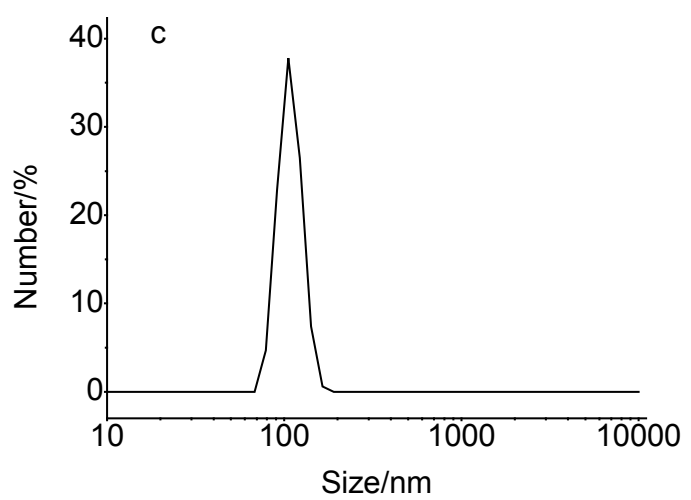

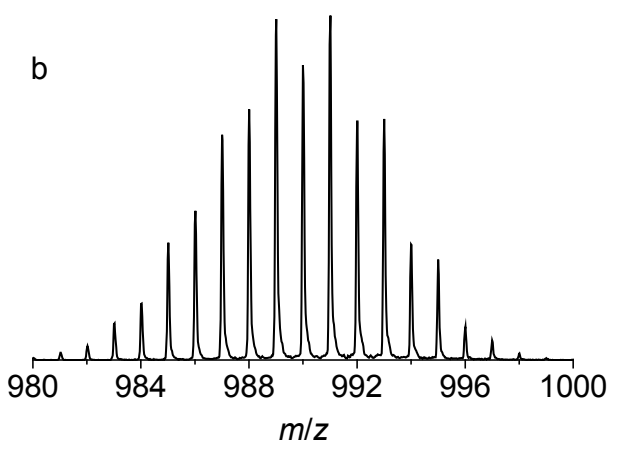

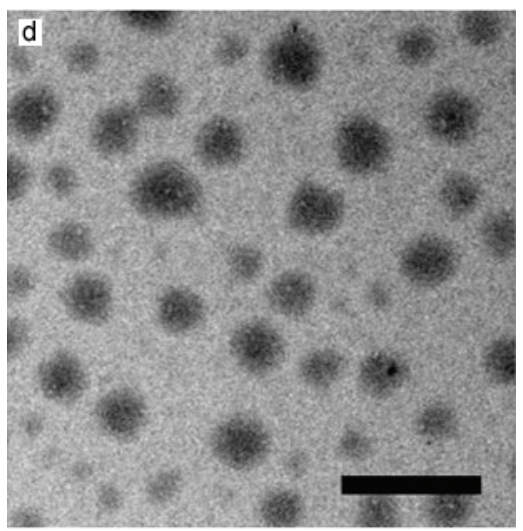

图 2 EGSe 和二氯化铂生成的配位组装体的结构与组装行为表征

Figure 2 Structure and assemble behavior of assembly formed by coordination between EGSe and $\mathrm{PtCl}_{2}$

(a) zoomed ${ }^{1} \mathrm{H}$ NMR spectrum $\left(298 \mathrm{~K}, 400 \mathrm{MHz}\right.$, solvent was $\mathrm{CDCl}_{3}$ ); (b) MALDI-TOF result; (c) DLS result (concentration of sample was $1 \mathrm{mmol} / \mathrm{L}$ ); (d) TEM Figure (concentration of sample was $1 \mathrm{mmol} / \mathrm{L}$, scale bar was $500 \mathrm{~nm}$ ).

如图 3 所示. 通过荧光探针法测定 EGSe-tMe 在水中的 临界聚集浓度 $(\mathrm{CAC})$ 为 $0.5 \mathrm{mmol} / \mathrm{L}$, 当 $\mathrm{EGSe}-\mathrm{tMe}$ 浓度 高于 $0.5 \mathrm{mmol} / \mathrm{L}$ 时即可在水溶液中发生聚集. 通过 DLS 法测定 EGSe-tMe 粒径约为 $190 \mathrm{~nm}$, 通过 TEM 观察组 装体的粒径与 DLS 法测定结果相符, 形成球状聚集体. 以上结果表明 EGSe-tMe 与 EGSe 在水相中具有相似的 组装行为.

随后, 将 EGSe-tMe 与二氯化铂进行配位, 对生成 的配位组装体 EGSe-tMe/Pt 进行表征, 结果如图 4 所示. $\mathrm{EGSe}-\mathrm{tMe} / \mathrm{Pt}$ 在水溶液中能够形成聚集体, DLS 法测得 其粒径约为 $156 \mathrm{~nm}$. TEM 下 EGSe-tMe/Pt 形成球状聚集 体, 粒径与 DLS 测试结果相符. MALDI-TOF 表征表明, 配位组装体并非简单地由 EGSe-tMe 和铂原子以化学计 量比 1:1 进行配位, 而是生成了多种不同的配位组装 体, 配位情况较为复杂. DLS 表征显示, 配位组装体 $\mathrm{EGSe}-\mathrm{tMe} / \mathrm{Pt}$ 在 $0.9 \%$ 的氯化钠溶液中的稳定性比 $\mathrm{EGSe} / \mathrm{Pt}$ 差(EGSe/Pt 的粒径在 $48 \mathrm{~h}$ 内维持在 $100 \mathrm{~nm}$ 左 右基本不变, 而 EGSe-tMe/Pt 的粒径在 $3 \mathrm{~h}$ 内就由 156 $\mathrm{nm}$ 增至 $1000 \mathrm{~nm}$ 以上). 此外, 在电子能谱表征中, EGSe-tMe 与铂配位后, 硒和铂的吸收峰位移均小于 $\mathrm{EGSe}$ 与铂配位后吸收峰的位移(图 S4). 综合以上证据,
我们认为 EGSe-tMe 和铂原子之间以一种较弱的配位作 用形成类似于超支化聚合物的聚集体结构.

\section{2 配位组装体的抗癌机制研究}

\subsubsection{EGSe-tMe 及其配位组装体的抗癌活性}

通过 MTT 细胞调亡实验对上文中制备的配位组装 体的抗癌活性进行研究, 结果如图 5 所示. 其中 HepG2 细胞为人肝癌细胞, L-02 细胞为人正常肝细胞. 在以 $250 \mu \mathrm{mol} / \mathrm{L}$ 的载药浓度载药 $24 \mathrm{~h}$ 后, 含硒分子 EGSe$\mathrm{tMe}$ 以及与二氯化铂的配位组装体 EGSe-tMe/Pt 均未表 现出明显的抗癌活性. 而配位组装体 $\mathrm{EGSe} / \mathrm{Pt}$ 具有较为 明显的选择性抗癌活性, 即对癌细胞杀伤作用较强而对 正常细胞毒副作用较小。通过对比可知, 含硒分子 EGSe 和 EGSe-tMe 均无明显的抗癌活性; 而配位组装体 $\mathrm{EGSe} / \mathrm{Pt}$ 表现出 EGSe-tMe/Pt 所不具备的显著的选择性 抗癌活性. 因此, 我们认为硒与铂之间紧密的配位作用 对配位组装体所表现出的选择性抗癌活性具有重要意 义。

\subsubsection{ROS 与配位组装体抗癌活性的关系}

采用能够特异性识别细胞内 ROS 的荧光探针 DCFH-DA，通过流式细胞仪对配位组装体在细胞内诱 
a
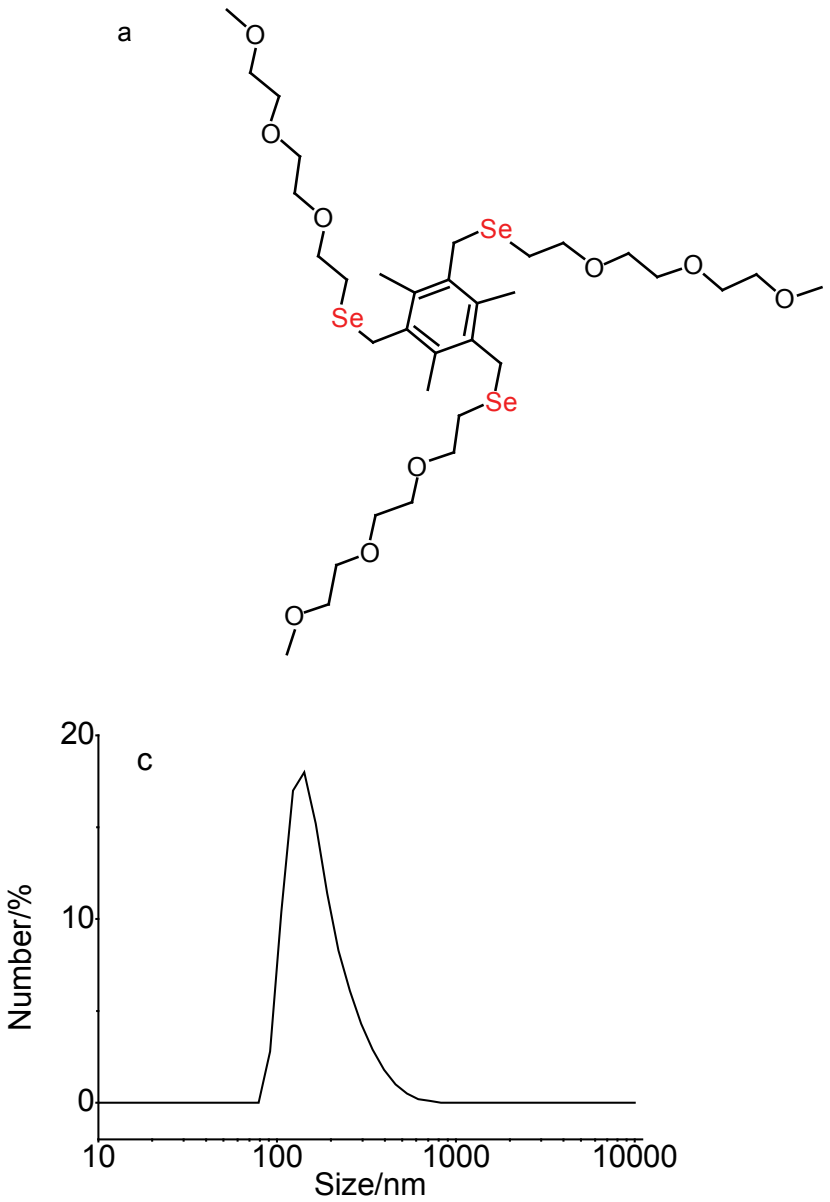
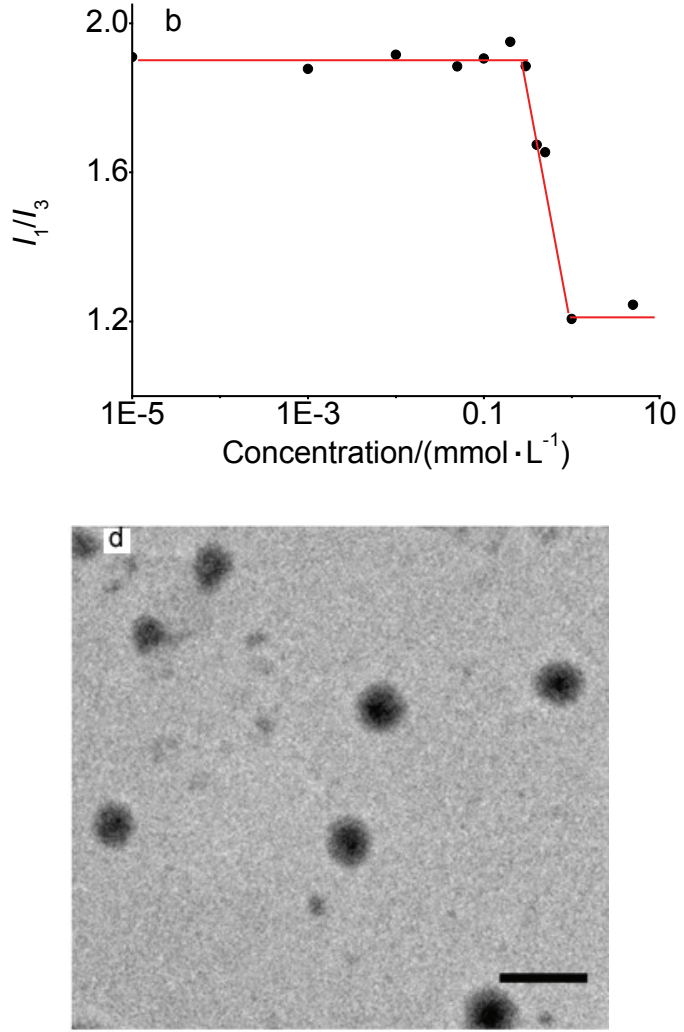

图 3 EGSe-tMe 的结构与组装行为表征

Figure 3 Structure and assemble behavior of EGSe-tMe

(a) chemical structure of EGSe-tMe; (b) CAC determined by fluorescent probe; (c) DLS result (concentration of sample was 1 mmol/L); (d) TEM Figure (concentration of sample was $1 \mathrm{mmol} / \mathrm{L}$, scale bar was $200 \mathrm{~nm}$ ).
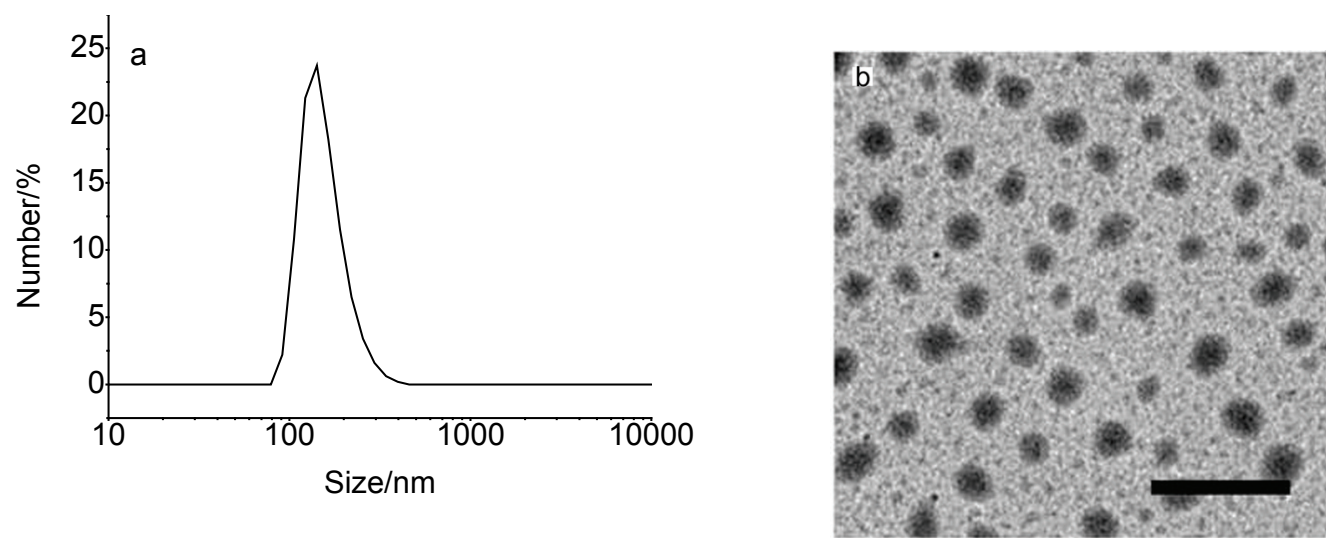

图 4 EGSe-tMe/Pt 的组装行为表征

Figure 4 Assemble behavior of EGSe-tMe/Pt

(a) DLS result (concentration of sample was $1 \mathrm{mmol} / \mathrm{L}$ ); (b) TEM Figure (concentration of sample was $1 \mathrm{mmol} / \mathrm{L}$, scale bar was $500 \mathrm{~nm}$ ).

导 ROS 产生的能力进行研究, 结果如图 6 所示, 荧光强 度越强表明细胞内 ROS 浓度越高. 在以 $250 \mu \mathrm{mol} / \mathrm{L}$ 的 载药浓度载药 $3 \mathrm{~h}$ 后, 加入 $\mathrm{EGSe} / \mathrm{Pt}$ 的实验组 $\mathrm{HepG} 2$ 细 胞内 ROS 浓度处于较高水平, 而 L-02 细胞内 ROS 浓度 相对较低, 表现出明显的选择性. 和 $\mathrm{EGSe} / \mathrm{Pt}$ 相比,
EGSe, EGSe-tMe 以及 EGSe-tMe/Pt 实验组中细胞内 ROS 的浓度均处于较低水平, 且在癌细胞和正常细胞 之间无明显的选择性. 将流式细胞仪的实验结果与上文 细胞调亡实验结果进行对比可知, EGSe/Pt 导致的 ROS 浓度的选择性与细胞调亡的选择性相吻合. 所以，我们 


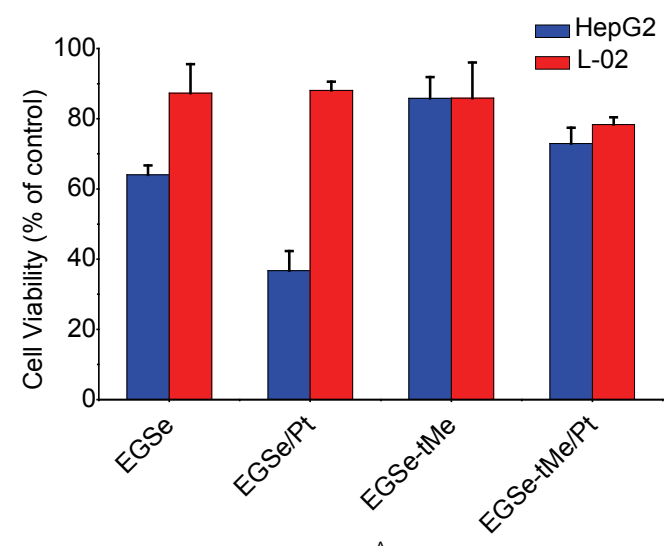

图 5 含硒分子及其配位组装体的细胞毒性实验结果

Figure 5 MTT results of selenium containing compounds and their coordinating assemblies

认为癌细胞和正常细胞内 ROS 的浓度差异对选择性抗 癌活性的产生起到非常重要的作用. 含硒配位组装体 $\mathrm{EGSe} / \mathrm{Pt}$ 在癌细胞中诱导产生高浓度的 ROS, 而在正常 细胞中诱导产生的 ROS 浓度较低, 从而实现选择性抗 癌效果.

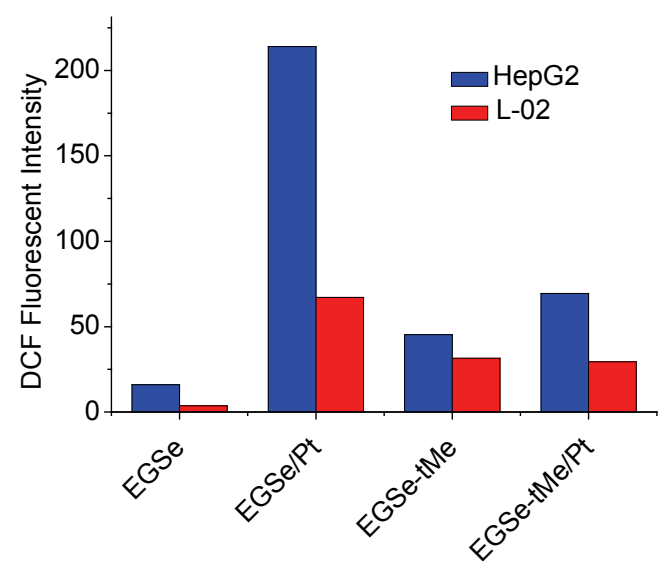

图 6 含硒配位组装体的流式细胞实验结果

Figure 6 Flow cytometry results of selenium containing coordinating assemblies

\subsubsection{ROS 产生机制的研究}

为了进一步说明体系催化产生 ROS 的机理, 我们 通过电子顺磁共振(ESR)对 ROS 的体外产生机制进行研 究, 结果如图 7 所示. 在加入 EGSe/Pt $(250 \mu \mathrm{mol} / \mathrm{L})$, $\mathrm{GSH}(1 \mathrm{mmol} / \mathrm{L})$ 和 $\mathrm{H}_{2} \mathrm{O}_{2}(100 \mu \mathrm{mol} / \mathrm{L})$ 的实验组中, 我们 可以观察到明显的羟基自由基信号. 若将 $\mathrm{EGSe} / \mathrm{Pt}$ 替换 为等浓度的 EGSe, 则不能观察到羟基自由基信号的生 成. 在不加入含硒分子, 只加入 $\mathrm{GSH}(1 \mathrm{mmol} / \mathrm{L})$ 和 $\mathrm{H}_{2} \mathrm{O}_{2}$ $(100 \mu \mathrm{mol} / \mathrm{L})$ 的实验组中, 也没有羟基自由基信号生成. 以上对比实验显示, $\mathrm{EGSe} / \mathrm{Pt}$ 能够在 $\mathrm{GSH}$ 和 $\mathrm{H}_{2} \mathrm{O}_{2}$ 的共同 作用下诱导 ROS 产生.
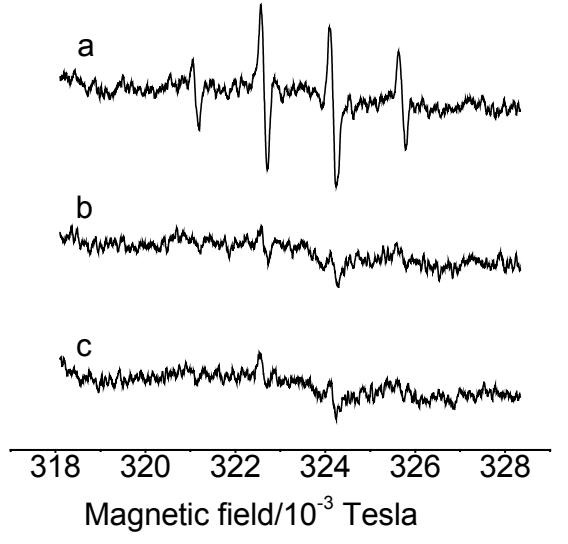

图 7 EGSe 及其配位组装体的 ESR 实验结果

Figure 7 ESR results of EGSe and its coordinating assembly (a) EGSe/Pt $(250 \mu \mathrm{mol} / \mathrm{L})+\mathrm{GSH}(1 \mathrm{mmol} / \mathrm{L})+\mathrm{H}_{2} \mathrm{O}_{2}(100 \mu \mathrm{mol} / \mathrm{L})$; (b) EGSe $(250 \mu \mathrm{mol} / \mathrm{L})+\mathrm{GSH}(1 \mathrm{mmol} / \mathrm{L})+\mathrm{H}_{2} \mathrm{O}_{2}(100 \mu \mathrm{mol} / \mathrm{L}) ;(\mathrm{c}) \mathrm{GSH}(1 \mathrm{mmol} / \mathrm{L})$ $+\mathrm{H}_{2} \mathrm{O}_{2}(100 \mu \mathrm{mol} / \mathrm{L})$.

\section{3 结论}

本文对具有含硒配位组装体中硒与铂的配位作用 和自组装行为进行研究. 证明了简单的铂类化合物能够 与含硒小分子发生配位，进而制备具有选择性抗癌活性 的配位组装体.

MTT 与流式细胞实验结果显示, 配位组装体的选 择性抗癌活性与细胞内 ROS 的浓度有较强的正相关性. 含硒配位组装体能够在 $\mathrm{GSH}, \mathrm{H}_{2} \mathrm{O}_{2}$ 的共同作用下诱导 ROS 产生, 组装体诱导 ROS 产生的能力在人肝癌细胞 和人正常肝细胞中具有差异, 从而表现出选择性抗癌活 性.

本文对一类基于硒铂配位的选择性抗癌体系进行 探索，拓展了含硒小分子和简单铂类化合物在抗癌领域 的应用, 为制备具有选择性抗癌活性的药物提供了新的 思路.

\section{4 实验部分}

荧光探针法测定含硒化合物 CAC: 分别配制浓度 为 $10^{-5}, 10^{-4}, 10^{-3}, 0.01,0.05,0.1,0.2,0.3,0.4,0.5,1,5$ $\mathrm{mmol} / \mathrm{L}$ 的 EGSe-tMe 溶液(溶剂为萠饱和水溶液)各 1 $\mathrm{mL}$, 使用荧光分光光度仪测定不同浓度 EGSe-tMe 溶液 在激发波长为 $335 \mathrm{~nm}$ 时的吸收曲线. 吸收曲线中可以 观测到萠的 4 个特征吸收峰, 其中由低波数向高波数方 向的第一个吸收峰的吸光度称为 $I_{1}$, 第三个吸收峰的吸 光度称为 $I_{3}$. 以 $I_{1} / I_{3}$ 对 EGSe-tMe 的浓度作图, 所得曲线 有两个拐点, 取两拐点横坐标的平均值为 EGSe-tMe 的 CAC 值.

TEM 测定含硒化合物组装形貌：将 $1 \mathrm{mmol} / \mathrm{L}$ EGSe-tMe 溶液滴加到碳支持膜上，吸附 $15 \mathrm{~min}$ 后用滤 纸吸走溶液并晾干. 由于硒本身是一种原子量较大的元 素，可以在 TEM 中成像，所以不用染色便可直接观察. 
DLS 测定含硒化合物组装体在盐溶液中的稳定性: 配制 $1 \mathrm{mmol} / \mathrm{L} \mathrm{EGSe} / \mathrm{Pt}$ 水溶液 $1 \mathrm{~mL}$, 放入比色血中, 用 DLS 测定组装体的初始粒径. 向组装体溶液中加入 9 $\mathrm{mg}$ 氯化钠粉末配制成 $0.9 \%$ 氯化钠水溶液, 每隔 $1 \mathrm{~h}$ 用 DLS 测定粒径.

细胞凋亡实验：人肝癌细胞(HepG2)和人正常肝细 胞(L-02)购自上海博谷生物科技有限公司. HepG2 细胞 用 DMEM 培养基培养, L-02 细胞用 RPMI1640 培养基培 养，培养时均需向培养基中加入 $10 \%$ 胎牛血清、 100 $\mathrm{U} / \mathrm{mL}$ 青霉素和 $100 \mu \mathrm{g} / \mathrm{mL}$ 链霉素. 培养环境为 $37{ }^{\circ} \mathrm{C}$, $5 \% \mathrm{CO}_{2}$.

进行细胞调亡测试时, 将 HepG2 细胞和 L-02 细胞 分别接种到 96 孔板上(每孔 $100 \mu \mathrm{L}$ 细胞悬液). 培养 $24 \mathrm{~h}$ 后移去培养基, 每孔加入 $100 \mu \mathrm{L} 250 \mu \mathrm{mol} / \mathrm{L}$ 待测药物 的培养基溶液. 培养 $24 \mathrm{~h}$ 后移去药物溶液, 每孔加入 $100 \mu \mathrm{L}$ 培养基和 $15 \mu \mathrm{L} \mathrm{MTT}$ 试剂. 孵育 $4 \mathrm{~h}$ 后移去培养 基, 每孔加入 $100 \mu \mathrm{L} \mathrm{DMSO}$, 在摇床上振荡 $20 \mathrm{~min}$ 后, 用酶标仪测定 $490 \mathrm{~nm}$ 下每孔溶液的吸光度, 通过与空 白对照组进行对比，计算细胞存活率.

细胞内 ROS 浓度测定: 将 HepG2 细胞和 L-02 细胞 分别接种到 24 孔板上(每孔 $1 \mathrm{~mL}$ 细胞悬液), 培养 $24 \mathrm{~h}$ 后移去培养基, 每孔加入 $1 \mathrm{~mL} 250 \mu \mathrm{mol} / \mathrm{L}$ 待测药物的 培养基溶液. $3 \mathrm{~h}$ 后移去药物溶液, 每孔加入 $1 \mathrm{~mL} 10$ $\mu \mathrm{mol} / \mathrm{L} \mathrm{DCFH}-\mathrm{DA}$ 的培养基溶液. 孵育 $20 \mathrm{~min}$ 后移去培 养基, 并用 PBS 洗涤 2 次, 加入 $100 \mu \mathrm{L}$ 胰蛋白酶, 将贴 壁细胞制成细胞悬液, 用流式细胞仪检测激发波长为 $488 \mathrm{~nm}$, 发射波长为 $525 \mathrm{~nm}$ 时的荧光强度, 该强度与细 胞内 ROS 浓度成正比.

\section{致谢 感谢张希院士的建议与讨论.}

\section{References}

[1] Kohanski, M. A.; Dwyer, D. J.; Hayete, B.; Lawrence, C. A.; Collins, J. J. Cell 2007, 130, 797.

[2] Liu, Y. Y.; Imlay, J. A. Science 2013, 339, 1210

[3] Keren, I.; Wu, Y. X.; Inocencio, J.; Mulcahy, L. R.; Lewis, K. Science 2013, 339, 1213.

[4] Valko, M.; Rhodes, C. J.; Moncol, J.; Izakovic, M.; Mazur, M. Chem.-Biol. Interact. 2006, 160, 1.
[5] Huang, X.; Liu, X. M.; Luo, Q.; Liu, J. Q.; Shen, J. C. Chem. Soc. Rev. 2011, 40, 1171.

[6] Tang, Y.; Zhou, L. P.; Li, J. X.; Luo, Q.; Huang, X.; Wu, P.; Wang, Y. G.; Xu, J. Y.; Shen, J. Q.; Liu, J. Q. Angew. Chem., Int. Ed. 2010, 49, 3920.

[7] Cao, W.; Xu, H. P. Chemistry 2013, 76, 291. (曹玮, 许华平, 化学 通报, 2013, 76, 291.)

[8] Rayman, M. P. P. Nutr. Soc. 2007, 64, 527

[9] Xu, H. B.; Zhang, L. P.; Fan, H. H.; Sun, J.; Wang, X. W.; Hu, S. Z. Acta Chim. Sinica 1989, 47, 901. (徐辉碧，张罗平，范华汉，孙杰， 汪新文, 胡松洲, 化学学报, 1989, 47, 901.)

[10] Wang, Z. G.; Ma, W. Y.; Zhang, C. N. Acta Chim. Sinica 1993, 51, 933. (王志光, 马维勇, 张椿年, 化学学报, 1993, 51,933.)

[11] Weekley, C. M.; Harris, H. H. Chem. Soc. Rev. 2013, 42, 8870.

[12] Ma, N.; Li, Y.; Xu, H. P.; Wang, Z. Q.; Zhang, X. J. Am. Chem. Soc. $\mathbf{2 0 1 0}, 132,442$.

[13] Zhang, X.; Xu, H. P.; Dong, Z. Y.; Wang, Y. P.; Liu, J. Q.; Shen, J. C. J. Am. Chem. Soc. 2004, 126, 10556.

[14] Cao, W.; Li, Y.; Yi, Y.; Ji, S. B.; Zeng, L. W.; Sun, Z. W.; Xu, H. P. Chem. Sci. 2012, 3, 3403.

[15] Xu, H. P.; Cao, W.; Zhang, X. Acc. Chem. Res. 2013, 46, 1647.

[16] Ren, H. F.; Wu, Y. T.; Ma, N.; Xu, H. P.; Zhang, X. Soft Matter 2012 , $8,1460$.

[17] Cao, W.; Zhang, X. L.; Miao, X. M.; Yang, Z. M.; Xu, H. P. Angew. Chem., Int. Ed. 2013, 52, 6233.

[18] Han, P.; Li, S. C.; Cao, W.; Li, Y.; Sun, Z. W.; Wang, Z. Q.; Xu, H. P. J. Mater. Chem. B 2013, $1,740$.

[19] Miao, X. M.; Cao, W.; Zheng, W. T.; Wang, J. Y.; Zhang, X. L; Gao, J.; Yang, C. B.; Kong, D. L.; Xu, H. P.; Wang, L.; Yang, Z. M. Angew. Chem., Int. Ed. 2013, 52, 7781 .

[20] Cao, W.; Gu, Y. W.; Meineck, M.; Xu, H. P. Chem. Asian J. 2014, 9, 48.

[21] Wang, L.; Cao, W.; Yi, Y.; Xu, H. P. Langmuir 2014, 30, 5628.

[22] Liu, J. Y.; Pang, Y.; Chen, J.; Huang, P.; Huang, W.; Zhu, X. Y.; Yan, D. Y. Biomaterials 2012, 33, 7765.

[23] Gu, X. Y.; Wang, C. Y.; Tong, Z. Acta Chim. Sinica 2013, 71, 1136. (顾晓瑜，王朝阳，童真，化学学报, 2013, 71, 1136.)

[24] Masahiko, O.; Hashimoto, K.; Shimada, J.; Sakagami, H. Anticancer Res. 2004, 24, 655.

[25] Allen, T. M. Nat. Rev. Cancer 2002, 2, 750.

[26] Gao, X. H.; Cui, Y. Y.; Levenson, R. M.; Chung, L. W.; Nie, S. M. Nat. Biotechnol. 2004, 22, 969.

[27] Brigger, I.; Duernet, C.; Couvreur, P. Adv. Drug Delivery Rev. 2002, 54,631 .

[28] Davis, M. E.; Zuckerman, J. E.; Choi, C. H.; Seligson, D.; Tolcher, A.; Alabi, C. A.; Yen, Y.; Heidel, J. D.; Ribas, A. Nature 2010, 464, 1067.

[29] Maeda, H.; Bharate, G. Y.; Daruwalla, J. Eur. J. Pharm. Biopharm. 2009, 71, 409.

[30] Nie, Y.; Zhang, Z. R.; Li, L.; Luo, K.; Ding, H.; Gu, Z. W. J. Mater. Sci.: Mater. Med. 2009, 20, 1849.

[31] Wang, Y. X.; Chen, P.; Shen, J. C. Biomaterials 2006, 27, 5292.

[32] Bae, Y. H.; Park, K. J. Controlled Release 2011, 153, 198.

[33] Zeng, L. W.; Li, Y.; Li, T. Y.; Cao, W.; Yi, Y.; Geng, W. J.; Sun, Z. W.; Xu, H. P. Chem. Asian J. 2014, 9, 2295. 